\title{
Relación de trombofilia con infertilidad y pronóstico reproductivo en técnicas de reproducción asistida de alta complejidad. Revisión sistemática
}

\author{
Juan Enrique Schwarze M., MSc ${ }^{1,2}$, Claudia Albornoz G., MSc. ${ }^{3}$ \\ ${ }^{1}$ Unidad Medicina Reproductiva, Clínica Monteblanco. ${ }^{2}$ Departamento Clínico de Obstetricia y Ginecología, Universidad \\ de Santiago. ${ }^{3}$ Servicio de Quemados, Hospital Asistencia Pública. Santiago, Chile.
}

\section{RESUMEN}

Cerca del $10 \%$ de las parejas no podrán conseguir un embarazo luego de estar expuesta a doce meses de coito sin protección, y deberán ser sometidas a algún tipo de técnica de reproducción asistida para conseguir un embarazo. En esta revisión sistemática buscamos determinar si hay evidencia que el tratamiento de trombofilia, endógena o exógena, en pacientes sometidas a técnicas de reproducción asistida mejora la tasa de embarazo. Encontramos un total de 29 publicaciones, de las que 6 fueron incluidos en el análisis. Los estudios observacionales muestran una asociación entre trombofilia e infertilidad, sin embargo, no existe evidencia para concluir si existe causalidad o no. No encontramos evidencia concluyente que el tratamiento profiláctico mejore la tasa de embarazo en mujeres infértiles sometidas a fecundación in vitro.

\section{PALABRAS CLAVE: Trombofilia, fecundación in vitro, revisión sistemática}

\section{SUMMARY}

Around $10 \%$ of couple will not achieve pregnancy after 12 months of exposure, and will undergo assisted reproductive technology. In this systematic review we seek to determine whether the treatment of trombophilia, both exogenous and endogenous, increase or not the clinical pregnancy rate. We found 29 articles, 6 were included in the analysis. We found evidence that there is an association of thrombophilia and infertility, but not that the treatment improve the clinical pregnancy rate in women undergoing in vitro fertilization.

\section{KEY WORDS: Thrombophilia, in vitro fertilization, systematic review}

\section{INTRODUCCIÓN}

Se estima que el $10 \%$ de las parejas no podrán conseguir un embarazo luego de estar expuestas a 12 meses de coito sin protección. De estas parejas, cerca de un tercio requerirán de técnicas de reproducción de alta complejidad (fecundación in vitro con y sin inyección intracitoplasmática de espermatozoides (1).
La tasa de embarazo clínico por ciclo iniciado, depende fundamentalmente de 2 factores: la edad de la mujer y la reserva ovárica, definida como el número de folículos primordiales remanentes en los ovarios. Es así como la tasa de embarazo clínico por ciclo varía entre un $40 \%$ en mujeres menores de 35 años y un $20 \%$ en mujeres de 40 y más años (2).

Asimismo, se acepta que la tasa de embarazo de cada mujer es igual en los tres primeros intentos, 
siendo peor en intentos posteriores (3). Una causa que podría explicar esta situación, es que luego de 3 ciclos sin embarazo, las mujeres sometidas a un cuarto intento tienen un peor pronóstico, ya que tendrían alguna condición desfavorable, como translocaciones genéticas, patología uterina y trombofilia (4).

Se entiende como trombofilia a la condición hereditaria o adquirida que constituye un factor de riesgo para hipercoagulabilidad y sus complicaciones asociadas como es el tromboembolismo. Se ha asociado a complicaciones perinatales (5) como aborto habitual (6), restricción del crecimiento intrauterino (7), desprendimiento prematuro de placenta normoinserta, preclampsia severa y síndrome de HELLP (8).

El objetivo de esta revisión es responder si en mujeres sometidas a técnicas de reproducción complejas, el tratamiento de una trombofilia, adquirida o hereditaria, mejora la tasa de embarazo. Para eso, realizamos una revisión sistemática de la literatura científica disponible, respecto al riesgo de infertilidad asociado a trombofilia, y si el tratamiento profiláctico mejora el pronóstico reproductivo, expresado como tasa de parto con al menos un recién nacido vivo por ciclo comenzado.

\section{MATERIAL Y MÉTODO}

Búsqueda de artículos y referencias en las bases de datos Medline, LILACS, DARE, HTA Database, Cochrane Database of Sistematic Reviews, sitios web de sociedad científicas (http://www.asrm. org/; http://www.eshre.com/), y listas de referencias de artículos identificados. La cobertura temporal fue hasta diciembre de 2010.

Los criterios de búsqueda fueron amplios y correspondieron a la presencia en cualquier campo de las palabras claves "thrombophilia" e "IVF". Los criterios de inclusión fueron publicaciones en inglés, castellano y alemán; estudios en seres humanos que hayan evaluado el riesgo de infertilidad en mujeres con trombofilia; estudios que evaluaran la efectividad del tratamiento profiláctico de trombofilia en mejorar la tasa de parto con al menos un recién nacido vivo. Los diseños de investigación incluidos fueron estudios analíticos controlados, estudios de correlación y análisis de estadísticas poblacionales. Los criterios de exclusión fueron estudios secundarios.

El análisis crítico formal fue según criterios predefinidos. En estudios de prevalencia, estudios de cohorte, estudios de casos y controles se consideró la presencia de ajuste de variables confusoras. En estudios experimentales se consideró el método de asignación, el enmascaramiento y las pérdidas de seguimiento.

Se confeccionó tablas con la descripción general de los estudios seleccionados, sus resultados, y condiciones de validez interna. Se entrega una síntesis cualitativa de los resultados del conjunto de los estudios.

\section{RESULTADOS}

Consideraciones metodológicas. Como se mencionó, las trombofilias corresponden a una serie de condiciones hereditarias y adquiridas que se asocian a un mayor riesgo de eventos trombóticos. Según progresa su estudio, se encuentran cada vez más mutaciones y condiciones trombofílicas, de modo que es imposible para un investigador determinar todas las causas trombofílicas en su estudio. Es así como la mayoría de estos pesquisan distintas condiciones trombofílicas, de modo que es muy difícil extrapolar los resultados.

Por razones éticas, no se puede realizar estudios clínicos en los que se induzca una condición de trombofilia en mujeres y determinar si esto causa o no infertilidad. Por lo tanto, la única fuente de información que tenemos es la de estudios observacionales. El modelo ideal sería realizar un estudio de cohorte, en el que se determinara la condición de tener o no trombofilia en mujeres antes de que busquen embarazo, y determinar la frecuencia de infertilidad, definida como la falta de embarazo luego de 12 meses de coito desprotegido (9). Ninguno de los estudios analizados tuvo este modelo.

La limitación principal de los estudios observacionales relacionados con trombofilia e infertilidad fue que no ajustaron todas las variables potencialmente confusoras, lo que hace que sus resultados no puedan considerarse en la mayoría de los casos concluyentes. Es importante en tal sentido analizar el conjunto de la evidencia disponible y la tendencia general y dirección de los resultados, antes que los hallazgos puntuales de alguna investigación. En el caso de los estudios experimentales, la principal limitación fue la falta de control de otras variables clínicas. Independientemente de otros factores presentes ya sea conocidos o desconocidos, el principal factor pronóstico de todo tratamiento de reproducción asistida es la calidad de los gametos femeninos (10). Esto queda más claro al considerar la tasa de embarazo en ciclos de ovodonación. En estos casos, la tasa de embarazo no varía significativamente según la edad de la ovorreceptora, y se comporta de acuerdo a la edad de la donante de ovocitos (11).

Estudios seleccionados y síntesis de resultados. 
Con los criterios de búsqueda descritos anteriormente encontramos un total de 29 publicaciones. Al aplicar los criterios de inclusión y de exclusión en los resúmenes, excluimos 23 publicaciones, seleccionando 6 artículos. Al revisar estas publicaciones, encontramos una que no habíamos identificado previamente, por lo que analizaremos 7 estudios: 5 observacionales (Tabla I) y 2 clínicos (Tabla II).

Estudios observacionales. En 2003, Martinelli y cols (12), publicaron un estudio de caso control, comparando la frecuencia de trombofilia (mutaciones en los genes de Factor $V$, protrombina, metiltetrahidrofolato reductasa, presencia de anticuerpos antifosfolípidos) en 234 mujeres sometidas a fecundación in vitro con o sin inyección intracitoplasmática de espermatozoides (162 mujeres que no lograron embarazo y 72 que embarazaron), con la frecuencia en 234 mujeres que concibieron espontáneamente; no encontraron diferencia estadísticamente significativa en los grupos. En 2004, Azem y cols (13), realizaron un estudio de caso control, en el que compararon la prevalencia de trombofilia en 45 mujeres con historia de cuatro o más ciclos fallidos de fecundación in vitro, y en 44 mujeres aparentemente sanas; la frecuencia de trombofilia fue mayor en mujeres con falla repetida de fertilizacion in vitro (43\%) que en los controles (18\%). En 2006, Coulam y cols (14), publicaron la prevalencia de mutaciones trombofílicas de 10 genes en 42 mujeres con historia de fracaso de transferencia embrionaria posfecundación in vitro, con la de 20 mujeres fértiles; más de tres mutaciones fueron encontradas en el $74 \%$ de las mujeres con falla implantatoria y en el $20 \%$ de los controles. En 2006, Qublan y cols (15), publicaron la frecuencia de mutaciones trombofílicas en 90 mujeres con al menos tres fallas de fecundación in vitro, con la de 90 mujeres que consiguieron embarazo luego del primer intento de fecundación in vitro y 100 mujeres que concibieron espontáneamente y sin antecedentes de aborto; la frecuencia de trombofilia en el primer grupo fue de $69 \%$, en el segundo de $26 \%$ $y$ en tercero de $25 \%$. En 2009 , Simur y cols (16), publicaron la frecuencia de trombofilia en 51 mujeres con tres fallas de fecundación in vitro con la frecuencia de trombofilia en 50 mujeres que concibieron espontáneamente; en el primer grupo el $63 \%$ tenía al menos una trombofilia, mientras que en el segundo grupo fue de $54 \%$.

Estudios experimentales. En 2003, Stern y cols (17), publicaron un estudio clínico en el que comparaban la tasa de implantación al administrar heparina, versus la tasa de implantación al administrar placebo, en mujeres con síndrome de antifosfolípi- dos y la transferencia de al menos 10 embriones sin conseguir embarazo; administraron a 296 mujeres heparina (5000 IU s.c. dos veces al día) y aspirina (100 mg diarios), mientras que 259 recibieron placebo, sin encontrar diferencias significativas entre ambos grupos en la tasa de implantación (7\% y $9 \%$, respectivamente). En 2008, Qublan y cols (18), publicaron un estudio clínico controlado en el que estudiaron la efectividad del tratamiento con heparina de bajo peso molecular en mujeres con trombofilia y falla recurrente de fecundación in vitro; 42 fueron aleatorizadas a recibir $40 \mathrm{mg} / \mathrm{día}$ de enoxaparina y 41 recibieron placebo, las del grupo tratado tuvieron tanto una mejor tasa de implantación $(21 \%$ y $6 \%$, respectivamente) como tasa de parto con al menos un recién nacido vivo (24\% y $3 \%$, respectivamente).

\section{DISCUSIÓN}

Al realizar la presente revisión, buscamos responder principalmente dos preguntas: ¿es trombofilia causa de infertilidad?, y en el caso que sea causa de infertilidad, ¿el tratamiento de trombofilia mejora la tasa de parto con al menos un recién nacido vivo? Cualquier análisis acerca de factores pronósticos de un tercer factor debe considerar la edad de la mujer, el número de embriones transferidos y el estadio de desarrollo embrionario al momento de la transferencia embrionaria. Ninguno de los estudios analizados hace este análisis, lo que en cierto grado le resta validez interna a sus conclusiones.

¿Es trombofilia causa de infertilidad?: Los estudios analizados no permiten responder esta pregunta, especialmente por dos razones. Salvo el estudio de Rudick y cols (19), todos los otros fueron determinaciones de prevalencia, de modo que no es posible determinar la temporalidad de la asociación trombofilia e infertilidad. Por otra parte, la población en la que se determinó la frecuencia de trombofilia también fue distinta; todos los estudios fueron en mujeres que no lograron embarazo. Azem y cols (20), ocuparon como población blanco a mujeres que tuvieron más de cuatro ciclos de fecundación in vitro; Coulam y cols (14) lo hicieron en mujeres a las que se les transfirió al menos 8 embriones de buena morfología; Qublan y cols (15) y Simur y cols (16), determinaron la frecuencia de trombofilia en mujeres con al menos tres fallas de fecundación in vitro, mientras que Vaquero y cols (4), determinaron la frecuencia de trombofilia en mujeres con al menos dos fallas de fecundación in vitro. En general la prevalencia de trombofilia varió entre un $43 \%$ y un $74 \%$. 
Tabla I

RESUMEN DE ESTUDIOS OBSERVACIONALES

\begin{tabular}{|c|c|c|c|c|c|}
\hline Autor/año & Tipo de estudio & Criterios de inclusión & Objetivo & Resultados & Conclusiones \\
\hline Martinelli/2003 & Cohorte prospectiva & $\begin{array}{l}\text { Mujeres caucásicas } \leq 40 \\
\text { años sometidas a FIV }\end{array}$ & $\begin{array}{l}\text { Comparar la prevalencia } \\
\text { de mutaciones en genes } \\
\text { de tombofilia entre mujeres } \\
\text { con falla FIV y controles } \\
\text { puérperas de embarazo } \\
\text { espontáneo. }\end{array}$ & $\begin{array}{l}\text { Sin diferencias entre los gru- } \\
\text { pos en frecuencia mutación } \\
\text { factor } V(8 / 162 \text { y } 5 / 234) \text {; } \\
\text { gen protrombina }(5 / 162 \text { y } \\
13 / 234) \text {; metiltetrahidrofolato } \\
\text { reductasa }(31 / 162 \text { y } 46 / 234) \text {. }\end{array}$ & $\begin{array}{l}\text { Trombofilia no predispone } \\
\text { a falla de la implantación } \\
\text { embrionaria }\end{array}$ \\
\hline Coulam/2006 & Prevalencia transversal & $\begin{array}{l}\text { No embarazo luego de la } \\
\text { transferencia de } \geq 8 \text { embrio- } \\
\text { nes en estadio de clivaje o } \\
\geq 4 \text { blastocistos }\end{array}$ & $\begin{array}{l}\text { Comparar la prevalencia de } \\
\text { mutaciones en genes de } \\
\text { tombofilia entre mujeres con } \\
\text { historia de falla en implanta- } \\
\text { ción }(n=42) \text { y controles con } \\
\text { fertilidad normal }(n=20) \text {. }\end{array}$ & $\begin{array}{l}\text { Sin diferencias en mutacio- } \\
\text { nes específicas. } \\
\text { Cuando la mutación del } \\
\text { Gen PAI- } 1 \text { es homocigota } \\
\text { se asocia con falla de la } \\
\text { implantación ( } 38 \% \text { casos } \\
\text { y } 10 \% \text { controles, } p=0.03 \text { ). } \\
\text { Las mujeres con falla en la } \\
\text { implantación tienen mayor } \\
\text { cantidad de mutaciones ho- } \\
\text { mocigotas que las controles } \\
\text { (78\% vs. } 20 \%, p=0.007) \text {. } \\
\text { Número total de muta- } \\
\text { ciones (heterocigotos y } \\
\text { homocigotos, fue mayor en } \\
\text { grupo control ( } 74 \text { vs. } 20 \% \text {, } \\
p=0.0004 \text { ) }\end{array}$ & $\begin{array}{l}\text { Existe asociación entre } \\
\text { presencia de genes de trom- } \\
\text { bofilia y falla de implantación } \\
\text { después de fecundación in } \\
\text { vitro }\end{array}$ \\
\hline Azem/2004 & Casos y controles & $\begin{array}{l}\text { Mujer con } 4 \text { o más ciclos } \\
\text { de falla fecundación in vitro, } \\
\text { con } \geq 3 \text { embriones de buena } \\
\text { calidad transferidos }\end{array}$ & $\begin{array}{l}\text { Determinar diferencias en } \\
\text { prevalencia de tombofilia } \\
\text { en casos }(n=45) \text { y controles } \\
(n=44) \text {. }\end{array}$ & $\begin{array}{l}\text { La incidencia global de } \\
\text { trombofilia en casos } 44 \% \text {, } \\
\text { controles } 18 \% \text { ( } p=0.012 ; \\
\text { OR } 3.6 ; \mathrm{Cl} 95 \% 1.25-10.6) \text {. } \\
\text { En el subgrupo de mujeres } \\
\text { con causa desconocida de } \\
\text { infertilidad, hubo } 61.9 \% \\
\text { trombofilia. Al comparar este } \\
\text { subgrupo con controles, se } \\
\text { encontró prevalencias de } \\
43 \% \text { vs. } 9 \%(p=0.002) .\end{array}$ & $\begin{array}{l}\text { Hay asociación entre trom- } \\
\text { bofilia y falla fecundación } \\
\text { in vitro }\end{array}$ \\
\hline Qublan/2006 & Casos y controles & $\begin{array}{l}\text { Mujeres con historia de falla } \\
\text { IVF al menos } 3 \text { veces }\end{array}$ & $\begin{array}{l}\text { Estudio de prevalencia de } \\
\text { trombofilia (factor } V \text { Leiden, } \\
\text { mutación protrombina, } \\
\text { MTHFR, mutación y déficit } \\
\text { de proteína S, C y antitrombi- } \\
\text { na III, anticoagulante lúpico } \\
\text { y anticardiolipina) en } 90 \text { mu- } \\
\text { jeres consecutivas con } \geq 3 \\
\text { fallas de fecundación in vitro } \\
\text { (casos); } 90 \text { mujeres con em- } \\
\text { barazo después de } 1 \text { ciclo de } \\
\text { fecundación in vitro (control } \\
\text { B); } 100 \text { mujeres con embara- } \\
\text { zos naturales y sin historia de } \\
\text { abortos (control C). }\end{array}$ & $\begin{array}{l}\text { Mayor prevalencia de } \\
\text { mutaciones del factor } \mathrm{V}, \\
\text { MTHFR y ac. antifosfolípidos } \\
\text { en el grupo de estudio. } 69 \% \\
\text { del grupo estudio tenían al } \\
\text { menos una trombofilia, vs. } \\
26 \% \text { y } 25 \% \text { grupos B y C } \\
\text { (p<0.01) }\end{array}$ & $\begin{array}{l}\text { La trombofilia juega un rol } \\
\text { importante en la falla de } \\
\text { fecundación in vitro. Mujeres } \\
\text { con falla fecundación in vitro } \\
\text { a repetición deberian ser } \\
\text { estudiadas para trombofilia. }\end{array}$ \\
\hline Simur/2009 & Caso y control & $\begin{array}{l}\text { Al menos tres ciclos fallidos } \\
\text { de FIV/TE }\end{array}$ & $\begin{array}{l}\text { Comparar la frecuencia de } \\
\text { alteraciones trombofílicas en } \\
\text { mujeres con fallas repe- } \\
\text { tidas de FIV (casos }=51 \text { ) y } \\
\text { en mujeres con embarazo } \\
\text { espontáneo (controles }=50 \text { ) }\end{array}$ & $\begin{array}{l}\text { Sin diferencia estadísti- } \\
\text { camente significativa en } \\
\text { frecuencia de trombofilia } \\
\text { entre casos } 32 / 51 \text { y contro- } \\
\text { les } 23 / 50 \text {. }\end{array}$ & $\begin{array}{l}\text { La mutación del Factor } V \text { de } \\
\text { Leiden, MTHFR y mutación } \\
\text { del gen de protrombina no } \\
\text { juegan un rol importante en } \\
\text { fallas consecutivas de FIV. }\end{array}$ \\
\hline
\end{tabular}

Asimismo, la población control también fue distinta. Rudick y cols (19) y Qublan y cols (15), determinaron la frecuencia de trombofilia en mujeres sometidas a fecundación in vitro que lograron embarazo; Simur y cols (16) y Vaquero y cols (4) $(46 \%$ y $40 \%$ de prevalencia, respectivamente), lo hicieron en mujeres aparentemente sanas, mientras que Coulam y cols (14) y Azem y cols (13), en mujeres sanas y embarazadas $(20 \%$ y $27 \%$ de prevalencia respectivamente).

De este modo, la evidencia analizada muestra que hay una mayor frecuencia de trombofilias en mujeres infértiles, y especialmente en aquellas que no consiguen embarazo luego de un procedimiento de fecundación in vitro. La evidencia no nos permite concluir que las trombofilias sean causa de infertilidad.

¿El tratamiento de trombofilia mejora la tasa de parto con al menos un recién nacido vivo?: Encontramos solamente dos estudios experimentales $(15,17)$ que probaron la eficacia de tratar pacientes con trombofilia antes de iniciar un ciclo de fecundación in vitro. Ambos estudios fueron doble ciego y aleatorizados. Si bien ambos investigadores ocuparon mujeres con al menos una trombofilia, Stern y cols (17) pusieron como criterio de inclusión el an- 
Tabla II

RESUMEN DE ESTUDIOS CLÍNICOS

\begin{tabular}{|c|c|c|c|c|c|}
\hline Autor/año & Criterio de inclusión & Intervención & Diseño & Resultados & Observaciones \\
\hline Stern/2003 & $\begin{array}{l}\text { Mujeres seropositi- } \\
\text { vas al menos para } \\
\text { ac. antifosfolípidos, } \\
\text { ac. antinucleares, } \\
\text { ac. anti- } 32-\text { glico- } \\
\text { proteina, que se } \\
\text { hubiesen tranferido } \\
\geq 10 \text { embriones bue- } \\
\text { na morfología sin } \\
\text { embarazo clínico }\end{array}$ & $\begin{array}{l}5000 \text { IU heparina } \\
\text { sc. cada } 12 \text { horas, } \\
\text { más } 100 \text { mg aspiri- } \\
\text { na po diarios }\end{array}$ & $\begin{array}{l}\text { Estudio prospectivo, } \\
\text { controlodo, aleato- } \\
\text { rizado, doble ciego. } \\
\text { Con entrecruzamiento } \\
\text { tratamiento/placebo al } \\
\text { transferir los embrio- } \\
\text { nes criopreservados. }\end{array}$ & $\begin{array}{l}\text { Sin cambios signifi- } \\
\text { cativos entre ambos } \\
\text { grupos en tasa de } \\
\text { implantación } 7 \% \\
(20 / 296) \text { en grupo } \\
\text { tratado y } 8 \%(22 / 259) \\
\text { grupo placebo; sin } \\
\text { diferencia en tasa } \\
\text { bebés vivos en grupo } \\
\text { tratado } 6 \% \text { (18/158) y } \\
7 \%(17 / 142) .\end{array}$ & $\begin{array}{l}\text { Cálculo de tamaño } \\
\text { muestral en función } \\
\text { de embriones no de } \\
\text { pacientes. } \\
\text { Diseño de entrecru- } \\
\text { zamiento introduce } \\
\text { sesgo de selección. } \\
\text { No se registró ca- } \\
\text { lidad embrionaria, } \\
\text { edad de la mujer } \\
\text { ni reserva ovárica. } \\
\text { No se hizo análisis } \\
\text { estratificado por } \\
\text { número de embrio- } \\
\text { nes transferidos, } \\
\text { estadio embrionario } \\
\text { de transferencia. }\end{array}$ \\
\hline
\end{tabular}
$\begin{array}{ll}\text { Qublan/2008 } & \begin{array}{l}\geq 3 \text { fecundación in } \\ \text { vitro sin embarazo } \\ \text { trombofilia }\end{array}\end{array}$
$40 \mathrm{mg} / \mathrm{d}$ enoxaparina sc desde el día de transferencia embrionaria
Estudio prospectivo, controlado, aleatorizado.

\author{
Al comparar con el \\ grupo placebo (42 \\ pacientes), el grupo \\ tratado (41 pacientes) \\ tuvo mejor tasa \\ de implantación \\ (21\% vs. $6 \%$, \\ $\mathrm{p}<0.001)$; tasa de \\ embarazo ( $31 \%$ vs. \\ $10 \%, \mathrm{p}<0.05)$; tasa \\ parto $(24 \%$ vs. $3 \%$, \\ $\mathrm{p}<0.05)$.
}

\author{
Sin cálculo de \\ tamaño muestral \\ No se registró ca- \\ lidad embrionaria, \\ edad de la mujer \\ ni reserva ovárica. \\ No se hizo análisis \\ estratificado por \\ número de embrio- \\ nes transferidos, \\ estadio embrionario \\ de transferencia.
}

tecedente de haber transferido al menos 10 embriones sin lograr embarazo, mientras que Qublan y cols (18) incluyeron mujeres con al menos tres ciclos de fecundación in vitro sin lograr embarazo. Las intervenciones fueron distintas. El grupo de Stern ocupó heparina subcutánea y aspirina oral, mientras que el de Qublan usó enaxaparina. Ninguno de los estudios consideró otros factores pronósticos como la edad de la mujer, el número de embriones transferidos y el estadio de desarrollo embrionario. Asimismo, los autores no señalan claramente como realizaron el enmascaramiento para los pacientes. La tasa de embarazo por transferencia embrionaria en los grupos tratados también fue distinta en ambos estudios: Stern y cols (17) encontraron en el grupo tratado un $15 \%$ de embarazo, similar al $18 \%$ del grupo placebo, mientras que Qublan y cols (18) encontraron un $31 \%$, mejor que el grupo placebo con un $10 \%$ de embarazos. Ninguna de las intervenciones consideró otros factores pronósticos, como edad de la mujer, número de embriones transferidos y estadio de desarrollo embrionario. Considerando esto, sumado a la heterogeneidad de los resultados, no es posible concluir que el tratamiento profiláctico de trombofilia mejora el pronóstico reproductivo de mujeres sometidas a fecundación in vitro.

\section{CONCLUSIONES}

Existe evidencia de estudios observacionales que sugieren que existe una asociación entre trombofilia e infertilidad. No existe evidencia para concluir si existe causalidad o no. No existe evidencia concluyente, que el tratamiento profiláctico mejore la tasa de embarazo en mujeres infértiles sometidas a fecundación in vitro. 


\section{BIBLIOGRAFÍA}

1. Schmidt L, Münster K. Infertility, involuntary infecundity, and the seeking of medical advice in industrialized countries 1970-1992: a review of concepts, measurements and results. Hum Reprod 1995;10(6):1407-18.

2. Zegers-Hochschild F, Schwarze JE, Crosby J, do Carmo M. Twenty years of Assisted Reproductive Technology (ART) in Latin America. J Brasileiro Reprod Assis 2011;15(2):15-30.

3. Sunderam S, Chang J, Flowers L, Kulkarni A, Sentelle $\mathrm{G}$, Jeng $\mathrm{G}$, et al. Assisted reproductive technology surveillance--United States, 2006. MMWR Surveill Summ 2009;58(5):1-25.

4. Vaquero $\mathrm{E}$, Lazzarin $\mathrm{N}$, Caserta $\mathrm{D}$, Valensise $\mathrm{H}$, Baldi $M$, Moscarini M, Arduini D. Diagnostic evaluation of women experiencing repeated in vitro fertilization failure. Eur J Obstet Gynecol Reprod Biol 2006;125(1):79-84.

5. Pabinger I. Thrombophilia and its impact on pregnancy. Thromb Res 2009;123 Suppl 3:S16-21.

6. Christiansen OB, Nielsen HS, Kolte AM. Future directions of failed implantation and recurrent miscarriage research. Reprod Biomed Online 2006;13(1):71-83.

7. Greer IA. Low molecular weight heparin for pregnancy complications? Thromb Res 2009;123 Suppl 3:S22-5.

8. Funai EF. Inherited thrombophilia and preeclampsia: is the evidence beginning to congeal? Am J Obstet Gynecol 2009;200(2):121-2.

9. Zegers-Hochschild F, Adamson GD, de Mouzon J, Ishihara O, Mansour R, Nygren K, et al. International Committee for Monitoring Assisted Reproductive Technology (ICMART) and the World Health Organization (WHO) revised glossary of ART terminology, 2009. Fertil Steril 2009;92(5):1520-4.

10. Scott RT, Opsahl MS, Leonardi MR, Neall GS, Illions $\mathrm{EH}$, Navot D. Life table analysis of pregnancy rates in a general infertility population relative to ovarian reserve and patient age. Hum Reprod 1995;10(7):1706-10.

11. Zegers-Hochschild F, Masoli D, Schwarze JE, laconelli A, Borges E, Pacheco IM. Reproductive performance in oocyte donors and their recipients: comparative analy- sis from implantation to birth and lactation. Fertil Steril 2010;93(7):2210-5.

12. Martinelli I, Taioli E, Ragni G, Levi-Setti P, Passamonti $\mathrm{SM}$, Battaglioli T, et al. Embryo implantation after assisted reproductive procedures and maternal thrombophilia. Haematologica 2003;88(7):789-93.

13. Azem F, Many A, Ben Ami I, Yovel I, Amit A, Lessing JB, Kupferminc MJ. Increased rates of thrombophilia in women with repeated IVF failures. Hum Reprod 2004;19(2):368-70.

14. Coulam CB, Jeyendran RS, Fishel LA, Roussev R. Multiple thrombophilic gene mutations are risk factors for implantation failure. Reprod Biomed Online 2006;12(3):322-7.

15. Qublan HS, Eid SS, Ababneh HA, Amarin ZO, Smadi AZ, Al-Khafaji FF, Khader YS. Acquired and inherited thrombophilia: implication in recurrent IVF and embryo transfer failure. Hum Reprod 2006;21(10):2694-8.

16. Simur A, Ozdemir S, Acar H, Colakoğlu MC, Görkemli $\mathrm{H}$, Balci $\mathrm{O}$, Nergis $\mathrm{S}$. Repeated in vitro fertilization failure and its relation with thrombophilia. Gynecol Obstet Invest 2009;67(2):109-12.

17. Stern C, Chamley L, Norris H, Hale L, Baker HW. A randomized, double-blind, placebo-controlled trial of heparin and aspirin for women with in vitro fertilization implantation failure and antiphospholipid or antinuclear antibodies. Fertil Steril 2003;80(2):376-83.

18. Qublan H, Amarin Z, Dabbas M, Farraj AE, Beni-Merei $\mathrm{Z}$, Al-Akash $\mathrm{H}$, et al. Low-molecular-weight heparin in the treatment of recurrent IVF-ET failure and thrombophilia: a prospective randomized placebo-controlled trial. Hum Fertil (Camb) 2008;11(4):246-53.

19. Rudick B, Su HI, Sammel MD, Kovalevsky G, Shaunik A, Barnhart K. Is factor V Leiden mutation a cause of in vitro fertilization failure? Fertil Steril 2009;92(4):1256-9.

20. Azem F, Samara N, Cohen T, Ben-Yosef D, Almog $\mathrm{B}$, Lessing JB, et al. Assessment of ovarian reserve following ovarian tissue banking and/or $\mathrm{GnRH}-\mathrm{a}$ co-treatment prior to chemotherapy in patients with Hodgkin's disease. J Assist Reprod Genet 2008;25(1112):535-8. 